\title{
Il parto extra-ospedaliero: un indagine quanti-qualitativa presso una casa maternità di Roma
}

\author{
Sofia Colaceci ${ }^{1}$, Roberta Vittoria Cicero ${ }^{2}$, Laura Reali ${ }^{3}$, Michele Grandolfo 4 , \\ Ivana Arena ${ }^{5}$, Sara Battaglia ${ }^{5}$, Daniela De Angelis ${ }^{5}$, Cristiana Romano ${ }^{6}$, Paola Mazza ${ }^{6}$, \\ Mariafrancesca Monsignore ${ }^{6}$, Stefania Olivieri $^{6}$, Federica Dellafiore ${ }^{7}$ \\ ${ }^{1}$ Ricercatrice, Facoltà dipartimentale di Medicina, Saint Camillus International University of Health and Medical Sciences
(UniCamillus), Roma; ${ }^{2}$ Dottoranda di ricerca, Dipartimento di Biomedicina e Prevenzione, Università degli Studi di Roma
Tor Vergata, Roma; ${ }^{3}$ Pediatra, Asl Roma 1; ${ }^{4}$ Epidemiologo, già Dirigente di ricerca, Istituto Superiore di Sanità, Roma;
${ }^{5}$ Ostetrica libero professionista, Zoè Casa maternità, Roma; ${ }^{6}$ Ostetrica, Corso di Laurea in Ostetricia, Università degli
Studi di RomaTor Vergata, Roma; ${ }^{7}$ Assegnista di ricerca, Università degli Studi di Pavia, Pavia
}

È stata condotta un'indagine quanti-qualitativa con l'obiettivo di descrivere gli esiti e il vissuto delle donne che hanno partorito in una realtà extra-ospedaliera di Roma tra il 2016 e il 2018. È stato effettuato uno studio osservazionale retrospettivo tramite la raccolta di dati clinico-assistenziali relativi alle 96 donne assistite nel triennio, di cui 9 trasferite in ospedale in travaglio. I principali outcome dei parti extra-ospedalieri ( $n=87$ ) sono stati: perineo integro nel 44,8\% dei casi, nessuna lacerazione di III o IV grado; $100 \%$ di clampaggio tardivo del funicolo, di cui il 26,4\% di lotus birth; prevalenza di allattamento esclusivo del 94,3\% a 7 giorni dal parto e del 93,3\% a 3 e 6 mesi.

La fase qualitativa è stata condotta su 15 donne tramite focus group e interviste in profondità. È emerso che la scelta di partorire in setting extra-ospedaliero è stata dettata prevalentemente dal desiderio di rispetto della fisiologia e dell'intimità della coppia o da un precedente vissuto negativo di parto ospedaliero.

\section{A quantitative-qualitative study was performed to describe outcomes and experiences of women who gave birth in an out-of-hospital setting in Rome, Italy, between 2016 and 2018. A retrospective observational study was carried out through the collection of clinical and healthcare data of 96 women assisted in these 3 years. Among them 9 were transferred to hospital during labour. The main outcomes of the out-of-hospital deliveries $(n=87)$ were: intact perineum and no third-or fourth-degree perineal tear in 44,8\% of cases, delayed cord clamping in 100\% of cases (of which $26.4 \%$ were lotus birth) and exclusive breastfeeding in $94.3 \%$ of cases at 7 days after delivery and $93.3 \%$ at 3 and 6 months. \\ The qualitative phase was conducted on 15 women and involved focus groups and in-depth interviews. It emerged that the choice to give birth in an out-of-hospital setting was mainly due to either the couple's respect for birth physiology and intimacy or a previous negative experience of hospital birth.}

\section{Introduzione}

Nei Paesi ad alto reddito il luogo del parto è prevalentemente l'ospedale. Tuttavia, per le gravidanze definite a basso rischio o fisiologiche, è possibile ricorrere al parto extra-ospedaliero, a domicilio o in casa maternità, qualora desiderato dalla coppia. Come ampiamente dimostrato dalla letteratura [1], nella popolazione a basso rischio ostetrico la scelta pianificata del luogo del parto ha un impatto poco significativo sugli esiti perinatali avversi. Inoltre, le donne a basso rischio che programmano di partorire in ambiente extra-ospedaliero risultano essere meno esposte a interventi invasivi (per esempio episiotomia ecc.) e a grave morbilità durante il travaglio e il parto [1].
Le prevalenze dei parti extra-ospedalieri sono modeste, attestandosi tra il 3 e il 5\% in Nuova Zelanda e tra l'1 e il 3\% in Giappone, Stati Uniti, Galles, Inghilterra, Scozia, Islanda e Svizzera [2-3]. Eccezion fatta per l'Olanda la cui prevalenza è stimata al 27\%, nei restanti Paesi europei non citati il dato è inferiore all'1\% [2-3] e, nello specifico, in Italia si attesta allo $0,1 \%$ [4] tant'è che l'interesse verso tale argomento potrebbe apparentemente non sembrare meritevole di attenzione. Tuttavia il parto extra-ospedaliero è stato menzionato in recenti linee di indirizzo sulle gravidanze a basso rischio [5] ed è stato oggetto di diverse normative regionali per il suo rimborso parziale.

Oltre che nelle consuete sale-parto ospe- daliere, il parto può essere espletato in centri nascita a gestione ostetrica situati all'interno o all'esterno dell'ospedale o in case maternità private gestite da ostetriche libero professioniste, nonché a domicilio [6]. In alcuni Paesi, come la Gran Bretagna, i centri nascita a gestione ostetrica afferiscono al sistema sanitario nazionale (SSN) e di conseguenza le prestazioni ivi erogate sono a carico dello stesso. In Italia le esperienze consolidate al riguardo sono concentrate nelle regioni del centro-nord: i centri nascita a gestione ostetrica afferenti al SSN sono tre (Genova, Torino e Firenze), mentre sono attivi diversi gruppi di ostetriche libero professioniste che assistono i parti presso case maternità private e/o a domicilio, le cui prestazioni sono a carico della coppia. In Piemonte, Emilia Romagna, Marche, Lazio e nelle province autonome di Bolzano e Trento è possibile richiedere un rimborso parziale, subordinato alla verifica dell'aderenza a precisi criteri di inclusione ed esclusione.

A oggi le uniche informazioni in termini di assistenza e di outcome dei parti-extraospedalieri nazionali derivano da uno studio su 1.099 donne che hanno optato per il parto extra-ospedaliero nel periodo 20142018 [2].

Sulla base di tali considerazioni, è stata condotta un'indagine quanti-qualitativa con l'obiettivo di descrivere gli esiti e il vissuto delle donne che hanno partorito in una realtà extra-ospedaliera di Roma.

\section{Obiettivi}

L'obiettivo primario dell'indagine è descrivere l'assistenza in gravidanza, travaglio, parto e puerperio e gli outcome di salute materno-neonatale riferiti ai parti extra-ospedalieri assistiti dalle ostetriche libero professioniste di una realtà romana. Obiettivo secondario è quello di esplorare 
il vissuto delle donne in merito alle motivazioni alla base della scelta di un parto extra-ospedaliero, al supporto del partner e della famiglia e alla soddisfazione dell'intero percorso.

\section{Metodi}

È stato condotto uno studio quanti-qualitativo relativamente ai parti assistiti tra il 2016 e il 2018 da un gruppo di ostetriche libero professioniste afferenti a una casa maternità privata di Roma, espletati presso la stessa o a domicilio. I requisiti professionali delle ostetriche, le indicazioni e le modalità per l'eventuale trasferimento di donna e/o neonato in ospedale e le procedure per l'eventuale attivazione del trasporto in urgenza/emergenza riferiti a questa casistica si attengono a quanto previsto dal DCA della regione Lazio del 23 dicembre 2016, n. U00395 [7]. Quest'ultimo prevede un rimborso parziale di 800 euro, corrispondente in media a un terzo della spesa sostenuta dalla coppia, ed è applicabile ai parti extra-ospedalieri in presenza di una gravidanza fisiologica e dell'aderenza a specifici criteri clinici.

Per la fase quantitativa è stato condotto uno studio osservazionale retrospettivo, utilizzando una scheda di raccolta dati, strutturata sulla base delle principali variabili presenti in letteratura sull'argomento $[2,8]$. Ogni scheda è stata compilata dalle ostetriche al termine della presa in carico di madre e bambino tramite i dati estratti dalle cartelle cliniche relativamente alle informazioni anamnestiche sull'assistenza in gravidanza e durante il travaglio, parto, post partum e puerperio. La scheda è stata completata dalle informazioni relative all'alimentazione infantile e alla soddisfazione dell'esperienza, acquisite tramite interviste telefoniche rivolte alle stesse donne che hanno avuto un parto extra-ospedaliero e condotte tra il $6^{\circ}$ e $7^{\circ}$ mese dallo stesso.

Successivamente è stata condotta la fase qualitativa finalizzata a rispondere all'obiettivo secondario dell'indagine. Tra i casi della fase quantitativa, è stato quindi selezionato un sottogruppo tramite campionamento di convenienza e sono stati condotti focus group e interviste in profondità, sino a saturazione dei dati, utilizzando una griglia di domande semistrutturate a cura di ricercatrici non coinvolte nell'assistenza clinica. I focus group e le interviste sono stati audioregistrati e trascritti parola per parola. I dati derivanti dalle trascrizioni sono stati codificati in categorie e sottocategorie e dunque analizzati tramite il metodo della long table analysis [9].

L'indagine è stata condotta previo consenso delle partecipanti e le informazioni so- no state trattate in forma anonima e conservate in accordo alle disposizioni vigenti in materia di protezione dei dati. L'indagine non è stata sottoposta all'approvazione di un comitato etico in quanto la normativa ne sancisce l'obbligo limitatamente agli studi sui farmaci [10].

\section{Risultati}

\section{Fase quantitativa}

Nel triennio 2016-2018 il gruppo di ostetriche selezionato ha assistito 96 donne di cui 1'83,3\% di nazionalità italiana. L'età media della popolazione in oggetto è di 34 anni (range 22-43), il 74,9\% è in possesso della laurea e il 54,2\% è costituito da pluripare (Tabella 1 ).

I trasferimenti in ospedale hanno coinvolto circa il 9,4\% della popolazione per: mancato inizio del travaglio $(n=2)$ o prolungamento dello stesso $(n=4)$, anomalie del battito cardiaco fetale ( $n=1)$, ipertensione $(n=1)$ e richiesta materna $(n=1)$. Di questi, 2 hanno interessato multipare e 7 nullipare. Il trasferimento è esitato in taglio cesareo per 3 donne, di cui 2 trasferite per prolungamento del travaglio e una per mancato inizio dello stesso.

In totale 87 donne hanno espletato un parto extra-ospedaliero (Tabella 2), di cui il $70,1 \%$ a domicilio e il $29,9 \%$ in casa maternità.

Per 4 parti a domicilio è stato richiesto l'intervento dell'ambulanza per casi di distocia di spalla, emorragia post partum e difficoltà respiratorie neonatali, ma in tutti vi è stata risoluzione in loco senza necessità di trasferimento in ospedale. In 2 casi le ostetriche hanno proceduto alla ventilazione del neonato con pallone autoespandibile e in un caso sono state effettuate compressioni toraciche, tuttavia l'intero campione dei nati ha raggiunto un punteggio di Apgar almeno pari a 7 entro il $10^{\circ}$ minuto. In nessun caso si sono verificati esiti materni o neonatali avversi.

Gravidanza, travaglio e parto. La quasi totalità della popolazione $(88,8 \%)$ ha partecipato agli incontri di accompagnamento alla nascita nell'ultima o precedente gravidanza. L'opportunità di usufruire della vasca da parto ha dato modo al $63,2 \%$ delle donne di farne uso in travaglio e al

\begin{tabular}{|c|c|c|}
\hline & Media & Range \\
\hline \multirow[t]{2}{*}{ Età (anni) } & 34 & $22-43$ \\
\hline & $\mathbf{N}$ & $\%$ \\
\hline \multicolumn{3}{|l|}{ Nazionalità } \\
\hline Italiana & 80 & 83,3 \\
\hline Straniera & 16 & 16,7 \\
\hline \multicolumn{3}{|l|}{ Titolo di studio } \\
\hline Media inferiore & 2 & 2,1 \\
\hline Media superiore & 21 & 21,9 \\
\hline Laurea & 71 & 74,9 \\
\hline Dato non presente & 2 & 2,1 \\
\hline \multicolumn{3}{|l|}{ Parità } \\
\hline Primipara & 44 & 45,8 \\
\hline Pluripara & 52 & 54,2 \\
\hline \multicolumn{3}{|l|}{ Incontri di accompagnamento alla nascita } \\
\hline Sì, nell'ultima gravidanza & 59 & 55,9 \\
\hline No, ma avevo partecipato nella precedente gravidanza & 27 & 32,9 \\
\hline No, mai & 10 & 12,2 \\
\hline \multicolumn{3}{|l|}{ Trasferimenti in ospedale } \\
\hline $\mathrm{Si}^{*}$ & 9 & 9,4 \\
\hline No & 87 & 90,6 \\
\hline \multicolumn{3}{|l|}{ Tipo di parto } \\
\hline Vaginale & 93 & 96,9 \\
\hline Taglio cesareo** & 3 & 3,1 \\
\hline \multicolumn{3}{|c|}{$\begin{array}{l}\text { * Motivi: mancato inizio (2) o prolungamento del travaglio (4), anomalie del battito cardiaco fetale (1). } \\
\text { ipertensione (1), richiesta materna (1). } \\
\text { ** Riferito a } 3 \text { trasferimenti, di cui } 1 \text { per mancato inizio e } 2 \text { per prolungamento del travaglio. }\end{array}$} \\
\hline
\end{tabular}


TABELLA 2. Parti extra-ospedalieri $(n=87)$

\begin{tabular}{|l|c|c|}
\hline Luogo del parto & N & $\%$ \\
\hline Domicilio & 61 & 70,1 \\
\hline Casa maternità & 26 & 29,9 \\
\hline
\end{tabular}

\section{Uso della vasca in travaglio e parto}

\begin{tabular}{|l|l|l|}
\hline Sì, in travaglio & 55 & 63,2 \\
\hline Sì, durante il parto & 29 & 33,3 \\
\hline
\end{tabular}

\section{Posizione al parto}

\begin{tabular}{|c|c|c|}
\hline Carponi & 44 & 50,6 \\
\hline Accovacciata & 19 & 21,8 \\
\hline Sul fianco & 10 & 11,5 \\
\hline In ginocchio & 6 & 6,9 \\
\hline In piedi & 4 & 4,6 \\
\hline Sdraiata & 2 & 2,3 \\
\hline Altra posizione & 2 & 2,3 \\
\hline \multicolumn{3}{|l|}{ Esiti perineali } \\
\hline Perineo integro & 39 & 44,8 \\
\hline Lacerazione di I grado & 29 & 33,3 \\
\hline Lacerazione di II grado & 18 & 20,7 \\
\hline Lacerazione di III o IV grado & 0 & 0,0 \\
\hline Episiotomia & 1 & 1,1 \\
\hline \multicolumn{3}{|l|}{ Manovra di Kristeller } \\
\hline Sì & 0 & 0,0 \\
\hline No & 87 & 100,0 \\
\hline \multicolumn{3}{|l|}{ Apgar $\geq 7$} \\
\hline $1^{\circ}$ minuto & 84 & 96,6 \\
\hline $5^{\circ}$ minuto & 86 & 98,9 \\
\hline $10^{\circ}$ minuto & 87 & 100,0 \\
\hline
\end{tabular}

\section{Manovre rianimatorie neonato}

\begin{tabular}{|l|c|c|}
\hline Sì, con manovre rianimatorie maggiori & 1 & 1,1 \\
\hline Sì, con pallone e maschera facciale o laringea & 2 & 2,3 \\
\hline $\begin{array}{l}\text { No/sì, stimolazione tattile (frizione, asciugatura, } \\
\text { massaggio) }\end{array}$ & 84 & 96,6 \\
\hline
\end{tabular}

\section{Richiesta ambulanza}

\begin{tabular}{|l|c|c|}
\hline Sì, utilizzata & 0 & 0,0 \\
\hline Sì, ma non utilizzata & 4 & 3,5 \\
\hline No & 83 & 96,5 \\
\hline
\end{tabular}

\section{Clampaggio del cordone}

Immediato

Dopo il $1^{\circ}$ minuto, prima del secondamento

\begin{tabular}{l|l}
0 & 0,0
\end{tabular}

Dopo il secondamento, entro 12 ore dal parto

\begin{tabular}{l|l}
0 & 0,0
\end{tabular}

Dopo il secondamento, dopo 12 ore dal parto

$\begin{array}{ll}25 & 28,7\end{array}$

Lotus birth integrale

$39 \quad 44,8$

\section{Contatto pelle-a-pelle immediatamente dopo il parto}

Sì

No

\begin{tabular}{c|c}
87 & 100,0 \\
\hline 0 & 0,0 \\
\hline
\end{tabular}

\section{Primo attacco al seno}

Entro mezz'ora dalla nascita

\begin{tabular}{|c|c|}
\hline 16 & 18,4 \\
\hline 63 & 72,4 \\
\hline 8 & 9,2 \\
\hline 0 & 0,0 \\
\hline
\end{tabular}

Dopo mezz'ora, entro 2 ore dalla nascita

Dopo 2 ore dalla nascita

Non allattamento

\section{Timing della visita pediatrica rispetto al parto}

Entro un'ora

\begin{tabular}{|l|c|c|}
\hline Tra 1 e 6 ore & 14 & 16,1 \\
\hline Tra 6 e 24 ore & 71 & 81,6 \\
\hline Dopo 24 ore & 2 & 2,3 \\
\hline
\end{tabular}

Visite ostetriche in puerperio

2-4

$5-7$

$\geq 8$

\begin{tabular}{|c|c|}
\hline 9 & 10,3 \\
\hline 70 & 80,5 \\
\hline 8 & 9,2 \\
\hline
\end{tabular}

\section{Problemi in puerperio}

Allattamento

Incontinenza urinaria

Lacerazione/sutura

Problemi di umore e sensazione di disagio

Problemi nella cura del neonato

\begin{tabular}{|c|c|}
\hline 24 & 27,6 \\
\hline 6 & 6,9 \\
\hline 5 & 5,7 \\
\hline 3 & 3,4 \\
\hline 3 & 3,4 \\
\hline
\end{tabular}

Prescrizione di formula artificiale entro i primi 3 giorni

$\mathrm{Si}^{*}$

No

\begin{tabular}{c|c}
\hline 2 & 2,3 \\
\hline 85 & 97,7
\end{tabular}

Modalità di alimentazione infantile a 7 giorni

Allattamento esclusivo

Allattamento predominante

Alimentazione complementare

Non allattamento

Modalità di alimentazione infantile a 1 mese ${ }^{* *}$

Allattamento esclusivo

Allattamento predominante

Alimentazione complementare

Non allattamento

Modalità di alimentazione infantile a 3 mesi**

\begin{tabular}{|l|c|c|}
\hline Allattamento esclusivo & 70 & 93,3 \\
\hline Allattamento predominante & 0 & 0,0 \\
\hline Alimentazione complementare & 3 & 4,0 \\
\hline Non allattamento & 2 & 2,7 \\
\hline
\end{tabular}

Modalità di alimentazione infantile a 6 mesi**

Allattamento esclusivo

Allattamento predominante

Alimentazione complementare

Non allattamento

\begin{tabular}{|c|c|}
\hline 82 & 94,3 \\
\hline 0 & 0,0 \\
\hline 5 & 5,7 \\
\hline 0 & 0,0 \\
\hline
\end{tabular}

Inizio del divezzamento**

Prima del $6^{\circ}$ mese completo

Dopo il $6^{\circ}$ mese completo

\begin{tabular}{|c|c|}
\hline 70 & 93,3 \\
\hline 0 & 0,0 \\
\hline 4 & 5,3 \\
\hline 1 & 1,4 \\
\hline
\end{tabular}

Tipologia di divezzamento**

Classico

Misto

Autosvezzamento

\begin{tabular}{|}
$\mid$\begin{tabular}{|c|c|}
\hline 70 & 93,3 \\
\hline 0 & 0,0 \\
\hline 3 & 4,0 \\
\hline 2 & 2,7 \\
\hline \\
5 & 6,7 \\
\hline 70 & 93,3 \\
\hline
\end{tabular}
\end{tabular}

\section{Esperienza di parto**}

Fortemente positiva/positiva

Ordinaria

Per alcuni aspetti positiva e per altri negativa

\begin{tabular}{|c|c|}
\hline 2 & 2,7 \\
\hline 18 & 24,0 \\
\hline 55 & 73,3 \\
\hline
\end{tabular}

Scelta del luogo del parto per eventuale parto successivo**

Setting extra-ospedaliero

Ospedale/clinica

\begin{tabular}{|c|c|}
\hline 75 & 100,0 \\
\hline 0 & 0,0
\end{tabular}

* Motivi: problemi di attacco, risolti entro una settimana.

**Dati riferiti alle donne che hanno partecipato all'intervista telefonica $(n=75)$. 
33,3\% di partorirvi. Le donne hanno partorito prevalentemente in posizione carponi $(50,6 \%)$ e accovacciata $(21,8 \%)$. Il $44,8 \%$ ha mantenuto integro il perineo, il $33,3 \%$ ha avuto una lacerazione di I grado, il $20,7 \%$ di II, mentre non ne è stata osservata alcuna di III o IV grado. Solo in un parto è stato necessario eseguire l'episiotomia. In nessun caso è stata praticata la manovra di Kristeller. Il clampaggio del funicolo è avvenuto nel $28,7 \%$ dei casi dopo il secondamento, entro 12 ore dal parto, nel $44,8 \%$ dopo 12 ore e nel $26,4 \%$ dei casi è stato effettuato il lotus birth integrale. Per quest'ultima pratica non è stato registrato alcun esito avverso di tipo infettivo.

Post partum e puerperio. A tutti i nati è stato garantito il contatto pelle-a-pelle immediatamente dopo il parto per più di 2 ore, supportando il primo attacco al seno solo quando fossero pronti e il $90,8 \%$ si è attaccato entro le 2 ore.

La prevalenza di allattamento esclusivo è stata del $94,3 \%$ a 7 giorni dal parto; 2 neonati hanno ricevuto una prescrizione di formula artificiale nei primi 3 giorni di vita per difficoltà di attacco superate entro una settimana.

La prima visita pediatrica è stata effettuata nel $97,7 \%$ dei casi entro 24 ore dalla nascita. Le ostetriche hanno effettuato prevalentemente tra le 5 e le 7 visite domiciliari in puerperio.

I problemi più frequenti riscontrati in puerperio hanno riguardato l'allattamento $(27,6 \%)$.

Le interviste telefoniche per l'integrazione delle informazioni hanno coinvolto 75 donne. Le restanti 12 sono state escluse per irreperibilità dopo almeno 3 tentativi di contatto $(n=7)$ o per rifiuto alla partecipazione $(n=5)$.

La prevalenza di allattamento esclusivo è stata del 93,3\% a 1, 3 e 6 mesi dal parto. Il $73,3 \%$ dei bambini ha praticato autosvezzamento.

L'esperienza del parto è stata definita positiva o fortemente positiva dal 93,3\% del campione, ordinaria dal 5,3\% e una donna ha evidenziato la coesistenza di aspetti positivi e negativi. L'intero campione sceglierebbe di nuovo il setting extra-ospedaliero per un eventuale parto successivo.

Fase qualitativa

Sono stati condotti 2 focus group, a cui hanno partecipato 10 donne, e 5 interviste singole in profondità. Dall'analisi dei dati sono emerse 5 categorie: motivazioni, supporto, emozioni in travaglio/parto, continuità assistenziale dopo il parto e cultura italiana sull'argomento (Tabella 3).

Motivazioni. La scelta di partorire in setting extra-ospedaliero è stata dettata pre-

TABELLA 3. Fase qualitativa - categorie e verbatim

\section{1) Motivazioni}

Rispetto della fisiologia.

"Avevo voglia di avere il tempo e lo spazio necessari per mettere al mondo mio figlio, senza troppe interferenze e in condizioni di fisiologia".

Precedente vissuto negativo di parto in ambiente ospedaliero.

"La cosa che mi ha sconvolta di più dell'esperienza in ospedale è non tanto l'esperienza oggettiva del parto che fa la mamma, quanto il fatto che non viene rispettato tanto l'incontro tra la mamma e il bambino e per me è ovvio che una donna che partorisce rimane attaccata al figlio sempre e subito".

\section{2) Supporto \\ Partner.}

"Il mio compagno poi mi ha appoggiato in tutto e per tutto, per me la priorità era anche che ci fosse lui, perché è una cosa che si fa in due, col parto non nasce solo la madre, ma una madre e un papà". Approccio graduale.

"Ho litigato tanto con mio marito perché all'inizio era totalmente contrario, anche perché lui ha avuto un altro figlio quindi aveva avuto l'esperienza dell'ospedale, poi l'ho portato a fare il corso preparto nella casa maternità e nel giro di pochissimo tempo si è convinto".

\section{Omissione.}

“...le famiglie, infatti all'inizio non le abbiamo proprio informate, anzi alla mia famiglia gliel'ho detto dopo il parto, perché sapevo che loro non sarebbero stati d'accordo, non avrebbero capito e non sarebbe stata una scelta condivisa".

\section{3) Emozioni in travaglio/parto}

"Anche la presenza del compagno è stata fondamentale, di supporto, per cui anche il sentire che eravamo io e lui... non sentivo altre interferenze, anche questo mi è piaciuto, cioè il fatto che l'ostetrica nella sua invisibilità, era invece molto presente".

\section{4) Continuità assistenziale dopo il parto}

"Le ostetriche però continuavano a venire a casa e per me questo è stato fondamentale, perché comunque ci hanno dato una mano enorme proprio nella gestione pratica della quotidianità per la bambina e per me, dandomi il tempo di riprendermi e di stare tranquilla".

\section{5) Cultura italiana}

\section{Mancanza di cultura .}

“La conoscenza c'è, ma non c'è la cultura del parto in casa, nel senso che la prima reazione è quella di chiusura per paura che sia troppo rischioso".

Alternative possibili sul luogo del parto.

"Poi c'è chi sceglie comunque di partorire in ospedale, ma è necessario che una donna sappia le alternative che ha".

"Quindi bisogna lavorare molto anche sull'autonomia delle donne, sul discorso di vivere quelle fasi di passaggio della vita della donna in maniera molto più naturale".

valentemente dal desiderio di rispetto della fisiologia e dei tempi del travaglio e dell'intimità della coppia. Per le pluripare la scelta è dipesa soprattutto da un precedente vissuto negativo di parto in ambiente ospedaliero.

Supporto. Sebbene decisivo per iniziare a chiedere informazioni sul parto extraospedaliero sia stato spesso il passaparola di altre donne che avevano vissuto direttamente l'esperienza, sulla scelta finale ha influito il punto di vista del partner. Talvolta, per quest'ultimo è stato necessario un approccio graduale al mondo del percorso nascita extra-ospedaliero. Le opinioni degli altri familiari (genitori, suoceri ecc.) hanno giocato un ruolo minore. Spesso è emersa 1'“omissione" della scelta del luogo del parto riservata ai familiari che non sarebbero stati d'accordo.

Emozioni in travaglio/parto. L'assistenza al travaglio/parto è stata descritta come rispettosa dell'intimità familiare e associata a emozioni positive.
Continuità assistenziale dopo il parto. La continuità assistenziale prestata in puerperio è risultata di primaria importanza per le donne poiché hanno beneficiato di un supporto costante in caso di dubbi sulla loro salute o su quella del bambino.

Cultura italiana. È emerso il tema della mancanza di cultura sul parto extra-ospedaliero, generalmente ritenuto dalla popolazione come meno sicuro di quello ospedaliero e associato a un maggior rischio di esiti avversi per la madre, ma soprattutto per il bambino. Infine, è emersa l'importanza prioritaria di informare le donne e le famiglie su tutte le alternative possibili sul luogo del parto e promuovere scelte consapevoli.

\section{Discussione e conclusioni}

La letteratura scientifica [11] conferma che le nascite extra-ospedaliere sono sicure in termini di esiti materno-neonatali laddove vi siano: una corretta identificazione dei fattori di rischio, la collaborazione del 
gruppo di ostetriche con un'équipe multidisciplinare di specialisti, in particolare ginecologi e pediatri e la possibilità di ricorrere al sistema di trasporto d'emergenza. In merito ai parti extra-ospedalieri del Lazio, il DCA n. U00395 del 2016 specifica le condizioni che necessitano del trasferimento in ospedale ed eventuali procedure da mettere in atto tempestivamente. A tal proposito, tutte le emergenze verificatesi nella casistica oggetto di indagine sono state risolte in setting extra-ospedaliero e i trasferimenti in condizioni di non urgenza/emergenza in ospedale hanno riguardato poco meno del $10 \%$ delle donne, dato ricompreso nei range riportati nella letteratura internazionale $[12,13]$. Il DCA n. U00395 del 2016 richiede infatti alle ostetriche specifici e rigorosi requisiti formativi e professionali, tra cui formazione continua sulla gestione delle emergenze e sulla rianimazione materna e neonatale.

Ulteriori outcome da menzionare sono tutti quelli derivati da un'assistenza fisiologica, tra cui i soddisfacenti esiti perineali, presumibilmente correlati al rispetto dei tempi di distensione perineale e della progressione della parte presentata e al mancato ricorso a procedure invasive come la manovra di Kristeller e l'episiotomia, effettuata solo in un caso ovvero per indicazione clinica.

In tutti i casi è stato effettuato un clampaggio ritardato del cordone. Inoltre, nonostante il dibattito sulla sicurezza del lotus birth sia ancora aperto $[14,15]$, una discreta proporzione di donne $(26,4 \%)$ ha optato per questa pratica, similmente a quanto riportato da Campiotti, et al. (28\%-36\%) [2]. La continuità assistenziale garantita dalle ostetriche fino al termine del puerperio assicura, tra le varie attività di care materno-infantile, anche un attento controllo del neonato per identificare tempestivamente i segni di una possibile infezione. In ogni modo, in nessun caso sono stati registrati eventi avversi neonatali di natura infettiva a esordio precoce o tardivo.

Per quanto riguarda l'alimentazione dei neonati, la prescrizione di formula artificiale a 3 giorni dal parto ha interessato un limitato numero di casi. Generalmente, il trend nei setting ospedalieri è maggiore, con prevalenze di allattamento esclusivo alla dimissione dai punti nascita subottimali, a eccezione degli ospedali certificati baby friendly [16]. Inoltre, si stima che nel 2017 almeno il 23\% dei bambini nati nel Lazio abbia ricevuto un cartellino di dimissione ospedaliero riportante uno spazio prestampato per la prescrizione della formula artificiale, indipendentemente dalla presenza o meno di un'indicazione clinica [17]. Altresì, è doveroso evidenziare che, sebbene i problemi di allattamento abbiano interessato una discreta percentuale di donne, la prevalenza di allattamento esclusivo è stata di oltre il $90 \%$ sino all'inizio del $6^{\circ}$ mese presumibilmente perché il sostegno prestato dalle ostetriche in puerperio ha consentito l'identificazione e la risoluzione tempestiva di tali problemi.

Infine, lo studio conferma che le donne che optano per il parto extra-ospedaliero hanno un caratteristico profilo socio-anagrafico: nello specifico, un titolo di studio elevato e un'età media maggiore rispetto alla popolazione che si rivolge alle strutture ospedaliere [2].

Come risulta dai dati qualitativi, il desiderio di ricevere un'assistenza fisiologica e una precedente esperienza negativa di parto ospedaliero sono i principali motivi addotti alla base della scelta di partorire in casa $o$ in casa maternità. La soddisfazione dell'esperienza del parto, oggigiorno uno dei principali indicatori della qualità dell'assistenza ricevuta [18], sia dai dati quantitativi che qualitativi, è molto elevata, contrariamente a una recente indagine riferita al contesto ospedaliero dalla quale emerge che circa il 40\% delle donne è soddisfatto solo in parte dell'assistenza ricevuta e il $6 \%$ per nulla [19].

I risultati dell'indagine non sono generalizzabili a causa della ridotta dimensione del campione e del coinvolgimento di un'unica realtà extra-ospedaliera, tuttavia hanno permesso di descrivere in modo esaustivo l'assistenza prestata e gli esiti materno-infantili. Sulla base di questo studio pilota, gli autori stanno attualmente implementando un sistema di raccolta dati per la sorveglianza attiva dei parti extra-ospedalieri della regione Lazio. Sarebbe auspicabile che lo stesso fosse successivamente esteso su scala nazionale con l'obiettivo di strutturare e implementare un midwifery data set dei parti extraospedalieri, utile per monitorare gli outcome di salute delle donne con gravidanze fisiologiche e dei loro bambini. Sull'esempio del Midwives Alliance of North America Statistics Project dataset adottato negli Stati Uniti [8], tale monitoraggio sarebbe un valido strumento per supportare il miglioramento continuo della qualità clinicoassistenziale erogata.

sofiacolaceci@gmail.com

La bibliografia è consultabile online. 
1. Scarf VL, Rossiter C, Vedam S et al. Maternal and perinatal outcomes by planned place of birth among women with low-risk pregnancies in high-income countries: A systematic review and meta-analysis. Midwifery 2018;62:240-55.

2. Campiotti M, Campi R, Zanetti M et al. Low-Risk Planned Out-of-Hospital Births: Characteristics and Perinatal Outcomes in Different Italian Birth Settings. Int J Environ Res Public Health 2020;17:2718.

3. Zielinski R, Ackerson K, Kane Low L. Planned home birth: Benefits; risks; and opportunities. Int. J. Women's Health 2015;7:361-77.

4. Ministero della Salute. Certificato di assistenza al parto (CeDAP). Analisi dell'evento nascita - Anno 2016. 2019.

5. Linee di indirizzo per la definizione e l'organizzazione dell'assistenza in autonomia da parte delle ostetriche alle gravidanze a basso rischio ostetrico (BRO) (2017). Disponibile al sito: http://www.salute.gov.it/imgs/C_17_pubblicazioni_2836_allegato.pdf

6. Rocca-Ihenacho L, Batinelli L, Thaels E et al. Midwifery Unit Standards. City University of London. 2018, versione italiana 2020. Disponibile al sito: http://www.midwiferyunitnetwork.org/wp-content/uploads/2020/02/ItalianMUStandards-Feb-2020.pdf

7. Decreto del Commissario ad Acta Regione Lazio, 23 dicembre 2016, n. U00395. Adozione del documento tecnico inerente "Protocollo assistenziale per il parto a domicilio in Centro Nascita e in Casa Maternità". Disponibile al sito: http://www.regione.lazio.it/binary/rl_sanita/tbl_normativa/SAN_DCA_U00395_23_12_2016.pdf

8. Cheyney M, Bovbjerg M, Everson C et al. Development and validation of a national data registry for midwife-led births: the Midwives Alliance of North America Statistics Project 2.0 dataset. J Midwifery Womens Health 2014;59:816.

9. Krueger RA, Casey MA. Focus Groups: A Practical Guide for Applied Research, 3rd ed. Thousand Oaks, CA: Sage Publications; 2000.

10. Legge 8 novembre 2012, n. 189. Conversione in legge, con modificazioni, del decreto-legge 13 settembre 2012 , n. 158, recante disposizioni urgenti per promuovere lo sviluppo del Paese mediante un più alto livello di tutela della salute. 11. Hutton EK, Reitsma A, Simioni J et al. Perinatal or neonatal mortality among women who intend at the onset of labour to give birth at home compared to women of low obstetrical risk who intend to give birth in hospital: A systematic review and meta-analyses. EClinicalMedicine 2019;14:59-70.

12. Blix E, Kumle M, Kjærgaard $\mathrm{H}$ et al. Transfer to hospital in planned home births: a systematic review. BMC Pregnancy Childbirth 2014;14:179.

13. Rossi AC, Prefumo F. Planned home versus planned hospital births in women at low-risk pregnancy: A systematic review with meta-analysis. Eur J Obstet Gynecol Reprod Biol 2018;222:102-8.

14. Monroe KK, Rubin A, Mychaliska KP, Skoczylas M, Burrows HL. Lotus Birth: A Case Series Report on Umbilical Nonseverance. Clin Pediatr (Phila)2019;58:88-94.

15. Steer-Massaro C. Neonatal Omphalitis After Lotus Birth. J Midwifery Womens Health 2020;65:271-5.

16. Marinelli A, Del Prete V, Finale E, Guala A, Pelullo CP, Attena F. Breastfeeding with and without the WHO/UNICEF baby-friendly hospital initiative: A cross-sectional survey. Medicine (Baltimore) 2019;98:e17737.

17. Colaceci S, Chapin EM, Zambri F, et al. Verba volant, scripta manent: breastfeeding information and health messages provided to parents in the neonatal discharge summary in the Lazio Region, Italy. Ann Ist Super Sanita 2020;56:142-9.

18. Anderson G, Zega M, D'Agostino F, et al. Meta-Synthesis of the Needs of Women Cared for by Midwives During Childbirth in Hospitals. J Obstet Gynecol Neonatal Nurs 2021;50:6-19.

19. Colaceci S, Corsi E, Berardi F et al. Soddisfazione materna dell'evento nascita: una web based survey. Professioni Infermieristiche. Prof Inferm 2020;73:181-7. 